\title{
Supporting Information: \\ Timing Correlations in Proteins Predict Functional Modules and Dynamic Allostery
}

\author{
Milo M. Lin \\ ${ }^{1}$ Green Center for Molecular, Computational, and Systems Biology \\ Department of Biophysics \\ University of Texas Southwestern Medical Center, Dallas, TX 75390 \\ ${ }^{2}$ Pitzer Center for Theoretical Chemistry, \\ University of California, Berkeley, CA 94720
}

E-mail: Milo.Lin@UTsouthwestern.edu 


\section{Backbone RMSD analysis}

The root-mean-square-deviations (RMSD) were calculated for backbone atoms of the catabolite activator protein complex using the trajectory generated by the 3 microsecond molecular dynamics simulations. The per-residue RMSD is shown in Fig. S1(top) and the protein-averaged RMSD is shown in Fig. S1(bottom). The backbone elements of the DNA binding region, especially the outer loops, experienced large conformational fluctuations. No noticeable movement of the long central helices were found. The regions of higher flexibility did not delineate the three functional modules, and did not elucidate the allosteric cyclicAMP binding site.
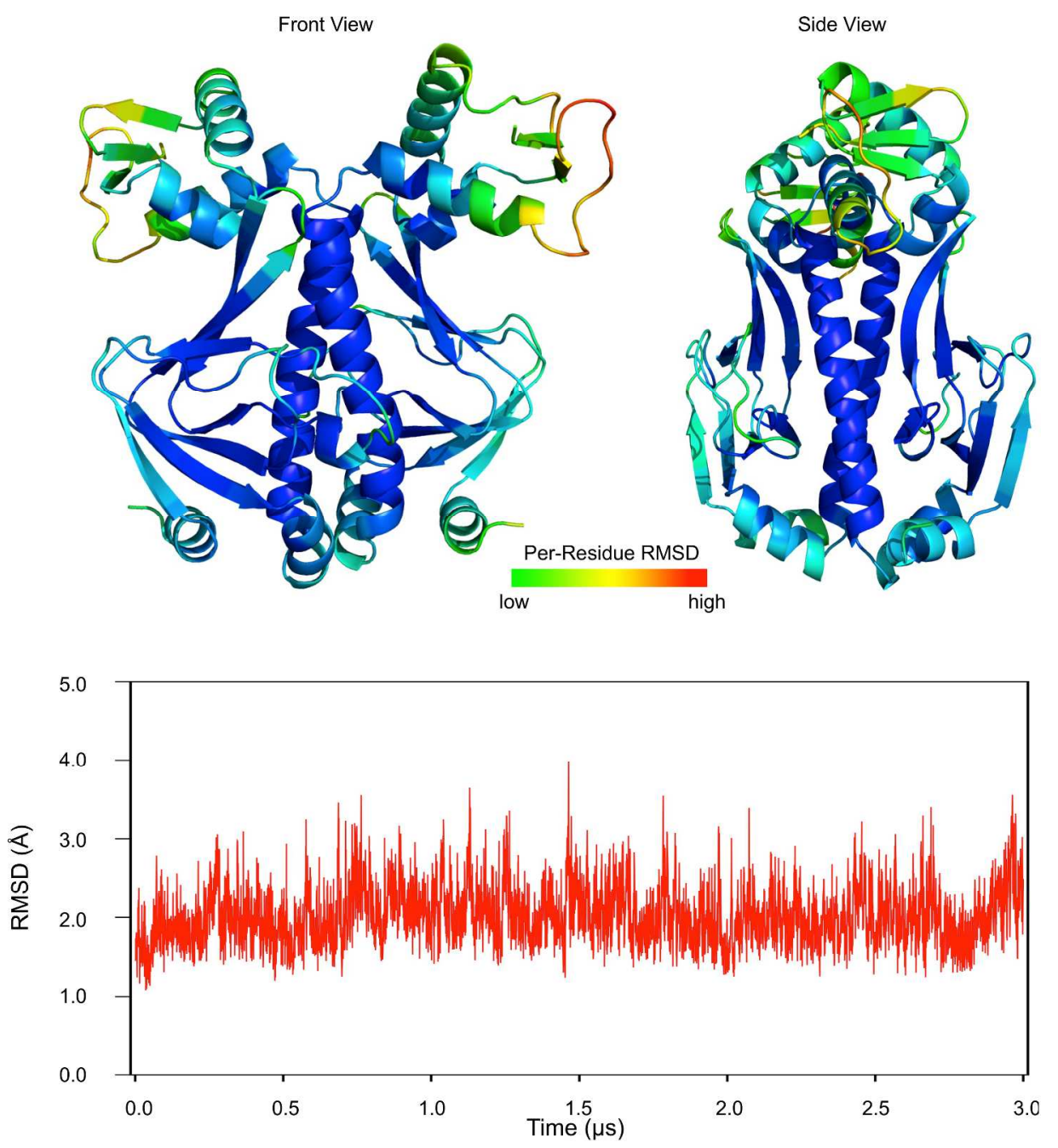

Fig. S1 Backbone RMSD of catabolite activator protein dimer. The per-residue RMSD (color coded; top) indicates that the surface residues of the loop regions in the active site are most mobile, and the RMSD averaged over the dimer indicates equilibration of backbone conformations (bottom). 
Principal Eigenmodes of the Mutual Information

A gap separates the first two principal eigenvalues of the dihedral mutual information matrix from the rest of the eigenvalue spectrum (see Fig. 4); their corresponding eigenvectors emphasize sub-populations of the dihedrals whose rotameric states are maximally correlated with each other. The eigenvectors are projected onto the catabolite activator protein structure (Fig. S2) in the same manner as the principal eigenvector of the conditional activity matrix (Fig. 5c). In contrast to the conditional activity, the principal eigenvectors are localized in space and orthogonal to the active site.

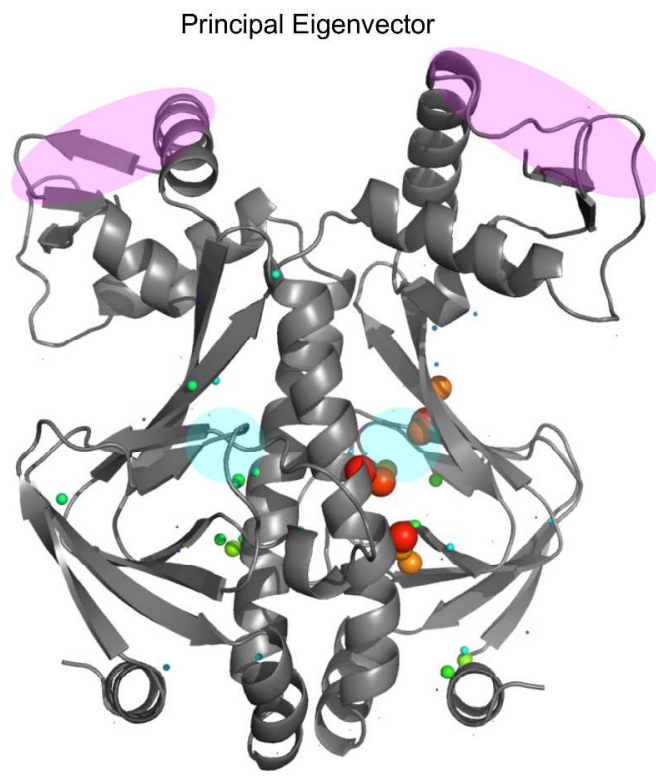

Allosteric Site

Active Site
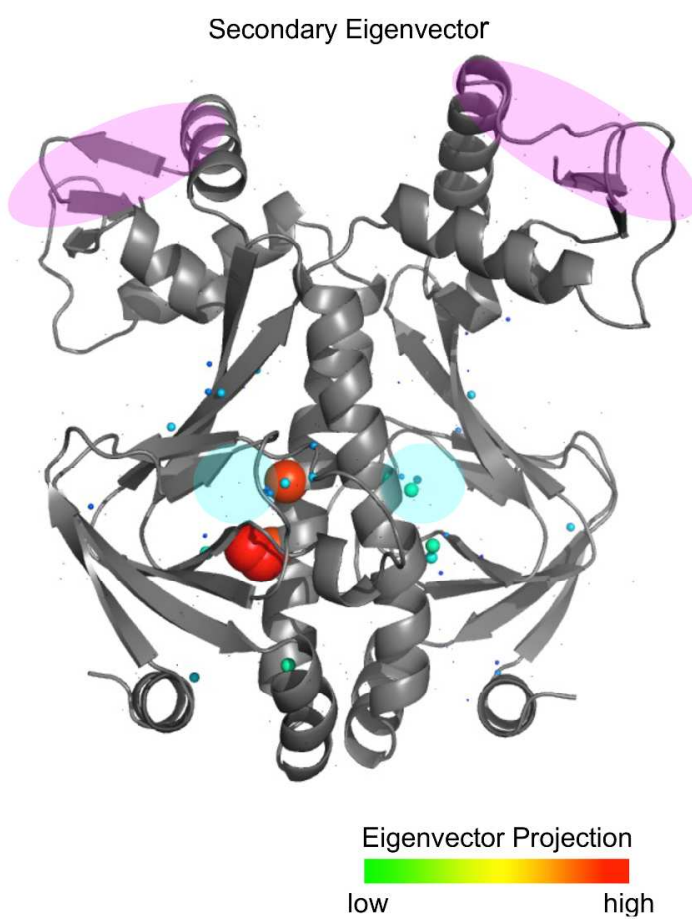

Fig. S2 Principal eigenvectors of the dihedral mutual information matrix in catabolite activator protein. The projection of the eigenvector onto the protein dimer is done as in Fig. 5 in the Text. Note that, in contrast to the long-range conditional activity shown in Fig. 5c, the collective modes corresponding to the two principal eigenvectors of the mutual information are local and do not include the active site. 
Low Frequency Elastic Modes

See upload movie files principal1.mpg, principal2.mpg, principal3.mpg, and principal4.mpg for the four lowest frequency (non-translational/rotational) normal modes calculated from the backbone atoms of the catabolite activator protein using the end structure of the molecular dynamics simulations. The pair-wise spring constants between every pair of backbone atoms was set to 0 if the distance between the atoms is greater than $1.0 \mathrm{~nm}$. Otherwise the spring constant was set proportional to a Gaussian function with mean 0 and standard deviation of $0.7 \mathrm{~nm}$. The matrix of pair-wise spring constants (Hessian) was diagonalized to yield the elastic modes. 